\title{
DISPERSION PROCESS: ROLE IN THE FORMULATION OF PARTICULATE DISPERSE SYSTEM OF POORLY SOLUBLE DRUGS
}

\author{
Bhaskar Rajveer*, Ola Monika, Patil PH \\ R. C. Patel Institute of Pharmaceutical Education \& Research, Karvand Naka, Shirpur, District Dhule, Maharashtra, INDIA 425405 \\ *Author for correspondence: Rajveer Bhaskar, Assistant Professor, Department of Pharmaceutics, R. C. Patel Institute of Pharmaceutical Education \& \\ Research, Karvand Naka, Shirpur, Distt, Dhule, Maharashtra, INDIA 425405, Email: bhaskar007_raj@rediffmail.com
}

Received 20 Dec 2012; Review Completed 09 Jan 2013; Accepted 09 Jan 2013, Available online 15 Jan 2013

\begin{abstract}
As significant number of products manufactured for personal care/health care, either in the final stage or at some stage of their production, dispersion of particulate materials dispersed into liquid or solid vehicles, often at high volume fraction. The vast array of cosmetics/personal care and pharmaceutical products reveal the importance of adequate dispersion. With active pharmaceutical ingredients possessing poor aqueous solubility, optimal dispersion is necessary for maximizing the bioavailability and uniformity of dose. Fine particulate dry powders always contain agglomerates that require deagglomeration and stabilization to obtain optimal dispersion. All particulate dispersion systems are inherently thermodynamically unstable. Unless properly stabilized, through the random motion of the particles over time, they will get aggregate due to the natural and dominant tendency to decrease the large specific surface area and excess surface energy. Adequate concentrations of wetting agents, de-agglomerating agents and stabilizers are required to produce stable particulate dispersions. The dispersion process is accomplished via three distinct steps: wetting the particulate material, de-agglomeration of the particles and stabilization of the same, and these steps should be performed in the correct order to get a stable product. Key words: de-agglomeration, stabilization, Ostwald ripening, particulate dispersion, aggregates.
\end{abstract}

\section{INTRODUCTION:}

The interest of pharmaceutical companies is increasing in the field of shelf life management, as it plays an important role in success of any formulation/ drug delivery technology. ${ }^{1-2}$ Drug delivery technologies which pose significant advantages over first generation commercial products provide an important means for staying competitive in today's growing and challenging business scenario. ${ }^{3-4}$ A superficial glance through the scientific and patent literature divulges that it is replete with examples in which decreasing the particle size of an active pharmaceutical ingredient (API) results in increased solubility and subsequent bioavailability. ${ }^{5}$ In the case of formulations intended for oral administration, poorly water soluble APIs may suffer from an inadequate rate and extent of drug absorption. Before formulating, the particle size reduction of API will significantly increase the specific surface area, saturation solubility, dissolution rate and finally bioavailability in gastrointestinal fluid. ${ }^{6}$ Therefore in the case APIs of BCS Class II and Class IV as per biopharmaceutical classification system, absorption is dissolution limited so decreasing the particle size may result in significant improvement in the rate and extent of drug absorption such that the bioavailability requirements of the drug candidates are met. ${ }^{7-9}$ Leading drug delivery technologies employing proprietary milling process that have produced nanotechnology integrated products such as Nanocrystal $^{\circledR}$ from Elan Drug Technologies, insoluble drug delivery (IDD ${ }^{\circledR}$ ) technology from Skye Pharma, Biorise $^{\circledR}$ technology from Eurand and NanoEdge ${ }^{\circledR}$ technology from Baxter Biopharma Solutions. ${ }^{10-}$ ${ }^{13}$ Nanocrystal technology has been the front runner that forms the technology platform for four marketed products in USA. Particle size can be decreased by various approaches but, subsequently in formulation, the API is usually dispersed in either aqueous or non-aqueous (c) 2011, JDDT. All Rights Reserved medium depending upon the pharmaceutical applications. Preparation of a stable formulation requires that a nonagglomerating and reproducible particle size distribution (PSD) should be achieved. This requires not only proper dispersion of the particles but also subsequent prevention of any re-agglomeration. Proper dispersion greatly enhances final product performance as well as maximizes efficiency and provides better product value from raw materials.
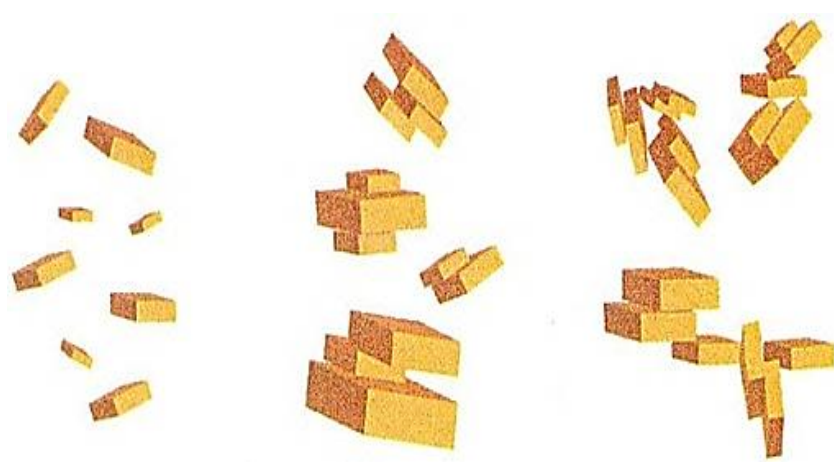

a. Primary particle b. Aggregates c. Agglomerates

Figure 1: Size nature of dry particles

Although having good formulation skills, experience and testing, problems can still sometimes be encountered in achieving stable, effective and elegant formulations containing nanoparticles. It is simply a matter of proper technique to disperse powders in liquids, based on an understanding of a few key principles. By considering each component of the disperse system in relation to the whole, logical choices can be made that will produce the anticipated product. All powders comprise three groups of particles: primary particles, aggregates and agglomerates. 
These three distinct species constitute what is known as the PSD of any nanoparticulate formulation.

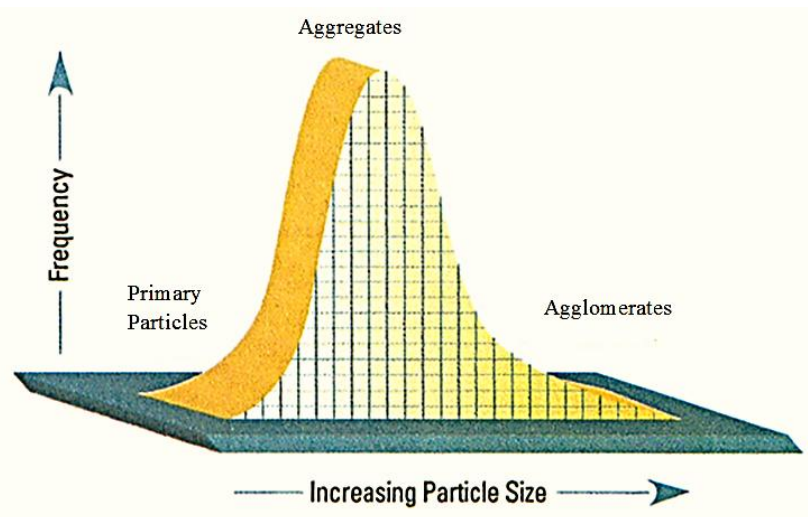

Figure 2: Relationship between different particle groups

The process of dispersion requires overcoming the various binding forces between particles by use of both physiochemical and mechanical means. The final dispersed state is accomplished via three distinct steps: wetting the solid surface, de-agglomeration of the particles and stabilization of the particles. It is crucial that these three steps be viewed as distinct and are performed in correct order. This is not to say that all three steps always require separate procedures but, the procedures must occur in sequence. In addition, the following factors will play an important role too.

$>$ Selection of the liquid medium for particulate dispersion.

$>$ Selection of the type and concentration of the various dispersion aids i.e. wetting agents, de-agglomerating agents and stabilizing agents.

$>$ Selection of the type and duration of the mechanical dispersing treatment.

The complete dispersion process is shown in figure 3 . Here each steps discussed separately in brief.

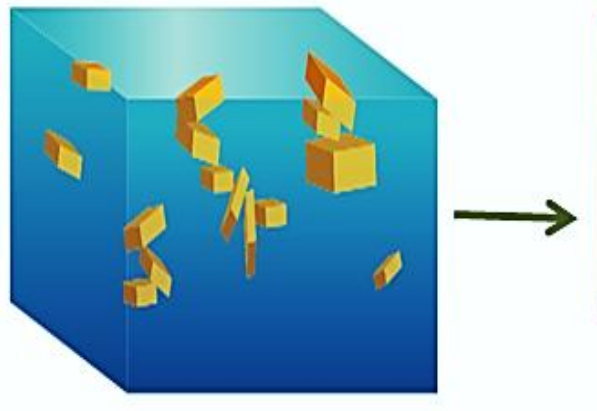

A. Spreading and wetting

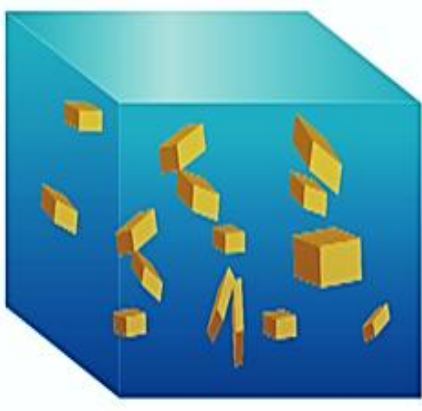

B. Deagglomeration

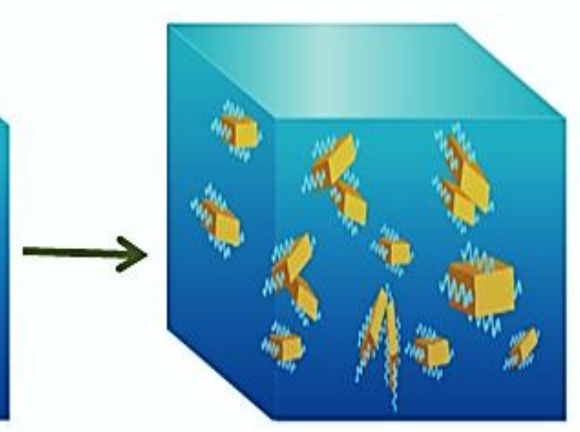

C. Steric stabilization

Figure 3: Process of the dispersion

\section{SPREADING AND WETTING:}

After adhesion the liquids spreads around the dry nanoparticles and wet them. Wetting is the ability of a liquid to maintain contact with a dry particle/surface, resulting from intermolecular interactions when the two are brought together. The degree of wetting (wettability) is determined by a force balance between adhesive and cohesive forces. Wetting involves contact of the liquid medium to the solid surface of nanoparticles, spreading of the liquid over the surface and finally penetration of the liquid into the spaces between agglomerated particles. Term 'lyophilic' is used for the surfaces which are easily wetted, while those are difficult to wet are termed 'lyophobic'; such surfaces require the use of a wetting agent. Many APIs falls in the latter category. The fundamental thermodynamic equations which govern adhesion and spreading are expressed by followings.

Spreading coefficient is expressed as

$$
\mathrm{S}=\gamma_{\mathrm{SG}}-\left(\gamma_{\mathrm{SL}}+\gamma_{\mathrm{LG}}\right)
$$

Young's equation is expressed as

$$
\gamma_{\mathrm{SG}}=\gamma_{\mathrm{SL}}+\gamma_{\mathrm{LG}} \cos \theta
$$

Combing the spreading coefficient with the Young's equation yields Young-Dupré equation.

$$
\mathrm{S}=\gamma_{\mathrm{LG}}(\cos \theta-1)
$$

Where $\gamma_{\mathrm{SG}}$ is the interfacial tension between the solid and the gas/vapor, $\gamma_{\mathrm{SL}}$ is the interfacial tension between the solid and liquid, $\gamma_{1 \mathrm{G}}$ is the interfacial tension between the liquid and gas/vapor, $\theta$ is the contact angle at the solid liquid interface and $S$ is spreading coefficient. A surface will be completely wet when $\gamma_{\mathrm{LG}}, \cos \theta$ tend to zero and $\mathrm{S}>$ 0 . If the value of spreading coefficient is less than 0 , there will be partial wetting.

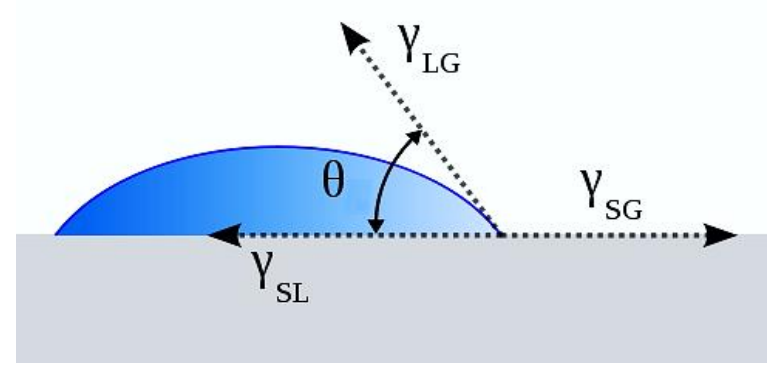

Figure 4: Forces acting on solid - liquid - gas (vapor) interface

To achieve complete wetting it is essential to add a wetting agent to the liquid. A wetting agent adsorbs at the liquid vapor interface, reducing the interfacial tension as well as the contact angle of the liquid at the surface of the particles. A wetting agent therefore termed as 'surface active agent' or 'surfactant'. The absolute minimum of the surfactant should be used to achieve wetting. Higher 
concentration of the surfactant will be increase the potential for foaming action rather wetting and this may lead the separation of the finer particles. An additional advantage of using the possible lowest concentration of the surfactant is to minimize any potential toxicity associated with

Table 1: Relationship between degree of wetting, contact angle and interacting forces

\begin{tabular}{|c|c|c|c|}
\hline \multirow[b]{2}{*}{ Degree of wetting } & \multirow[b]{2}{*}{ Contact angle } & \multicolumn{2}{|c|}{ Strength of } \\
\hline & & $\begin{array}{l}\text { Cohesive forces at solid- } \\
\text { liquid interface }\end{array}$ & $\begin{array}{c}\text { Adhesive forces at liquid- } \\
\text { liquid interface }\end{array}$ \\
\hline \multirow{2}{*}{ Perfect wetting } & \multirow{2}{*}{$\theta=0$} & Strong & Weak \\
\hline & & Strong & Strong \\
\hline High wettability & $0<\theta<90$ & Weak & Weak \\
\hline Low wettability & $90 \leq \theta<180$ & Weak & Strong \\
\hline Perfectly non wetting & $\theta=180$ & Weak & Strong \\
\hline
\end{tabular}

It is also possible to pre-wet a surface without using a surfactant. Any liquid that has contact angle lower than water will be sufficient. For example ethanol and propylene glycol can be used at the place of water as wetting liquid. Liquid compounds having humectant nature (Glycerin,) will also work and should be considered to enhance wettability. The consequence of not achieving complete wetting is to affect the suspension properties, especially the stability. It is equally important to realize that if a solid wets its own (i.e. lyophilic particles) then a wetting agent is not required. In fact, the use of one may be makes the subsequent deagglomeration step more complex, which finally leads to an unstable system.

\section{DEAGGLOMERATION:}

When the liquid medium sufficiently wet the solid particles, the liquid starts to penetrate into the pores and capillaries between to particles and this tends to them arrange separately in the liquid medium. The rate of penetration is an important factor. It is desirable that this be as high as possible. An indication of the major variables which affects the penetration rate is expressed by the Washburn equation.

$$
\mathrm{L}^{2}=\left(\mathrm{r} \text { t } \gamma_{\mathrm{LG}} / 4 \eta\right)
$$

Where $\mathrm{L}$ denotes the depth of penetration into a pore of radius (r) in time $(t)$ and $(\eta)$ is the viscosity of the liquid medium. In general, most of the wetting process (adhesion, spreading and penetration) is more spontaneous the lower the contact angle $(\theta)$ and higher the liquid - gas/vapor interfacial tension $\left(\gamma_{\mathrm{LG}}\right)$. Mostly all the surfactants affects both of these parameters, rendering the selection process of best agent for any system more difficult.

The process of separation of particles from each other once they are wetted is termed as deagglomeration. A deagglomerating agent adsorbs only at the solid - liquid interface. Such substances are not 'surface active'. Their job is to chemically aid separation of the agglomerated particles by increasing the electrostatic forces of repulsion between the particles. This allows further liquid penetration into the inter-particle spaces which ultimately enhance the separation process. For easily wetted material, this enhanced penetration of liquid into the void spaces between the particles may provide adequate force alone to bring about complete dispersion. The selection of the suitable deagglomerating agent requires consideration of both the particle's surface chemistry and the dispersion medium conditions. For example taking water as the liquid medium, pH and electrolyte concentration also considered. Following initial wetting of the particles some mechanical agitation is needed. The degree of turbulence and shear vary considerably with the type of operation and design of the equipment. Essentially there are two processing methods, first is high shear mills in which efficiency depends on the formulation viscosity and secondly high impact mills in which efficiency depends on the size of the grinding media. A detailed overview on processing methods for the formulation of nanoemulsion/nanosuspensionand particulate dispersion systems can be find the different scholarly review and research articles. If however, the various steps previously discussed are followed, then the minimum of mechanical agitation will be adequate. Excess mechanical energy increases the thermal energy, and together they can be lead to re-agglomeration, alteration in PSD and subsequently the total available surface area. In addition, it is also possible to change in the surface chemistry. All of the abovementioned impinges on the specific properties of the suspension.

\section{STABILIZATION:}

Stabilization is the act of keeping the particles apart once they are wetted and deagglomerated. Essentially, it is the last step of the dispersion process. If completed too early, it just stabilizes agglomerates only. Hence, the order of addition of the ingredients is always of concern. Stabilization is usually accomplished via steric forces (adsorbed layers), electrostatic forces (surface charges) or a combination of both. Steric stabilization is preferred if the suspension is formulated in non-aqueous medium. 'Thickeners' are often used to enhance the viscosity of the liquid. But it cannot be a substitute of true stabilization. Increasing the viscosity simply retard the sedimentation rate of the larger and/or denser particles. In any case thickeners should be incorporated after the stabilization step. As previously outlined the dispersion process is a series of orderly events, each reliant on the previous one. The performance of any system including pharmaceutical formulations is directly associated to this process. As 
Bhaskar et al

Journal of Drug Delivery \& Therapeutics; 2013, 3(1), 93-97

mentioned earlier, proper dispersion can greatly enhance the appearance and performance. As an example, the improved oral bioavailability of Elan Drug Delivery's NanoCrystal technology formulation approach is based on the increased surface area of the nano milled APIs giving rise to increased dissolution rate of the NanoCrystalCollidalDispersion ${ }^{\mathrm{TM}}$. However it is necessary to keep these nnaoparticles distinct in order to benefit from the enhanced surface area. This can be achieved by the use of both steric and electrostatic stabilization. This technology has been commercially validated and successfully used in many marketed products (i.e. First Horizon Pharmaceutical's Triglide ${ }^{\mathrm{TM}}$, Abbott's Tricor ${ }^{\circledR}$, PAR Pharmaceutical's MEGACE ${ }^{\circledR}$ ES, Merck's EMEND ${ }^{\circledR}$ and Wyeth's RAPAMUNE ${ }^{\circledR}$ ). Finally wet suspension can 'age' on standing/storage or during shelf life, due to Ostwald ripening. Ostwald ripening is responsible for destabilizing all types of dispersions (suspensions, emulsions, foams, nanosuspension, nanoemulsions etc.).

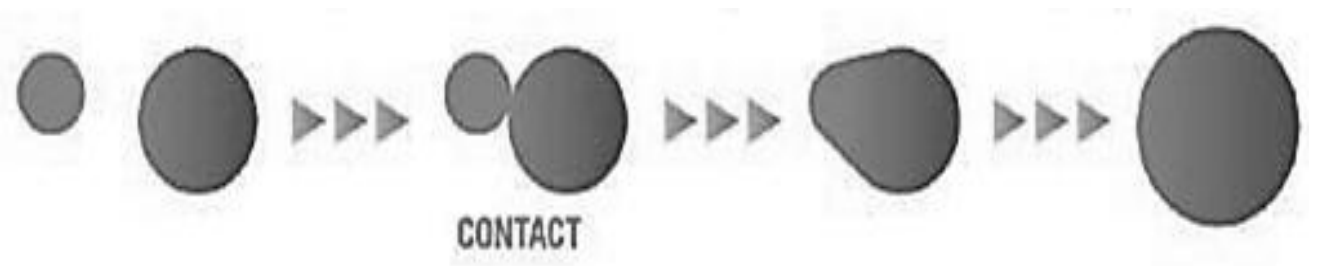

A. Un-stabilized particles: on contact, small particles with higher radial pressure feeds in larger particles

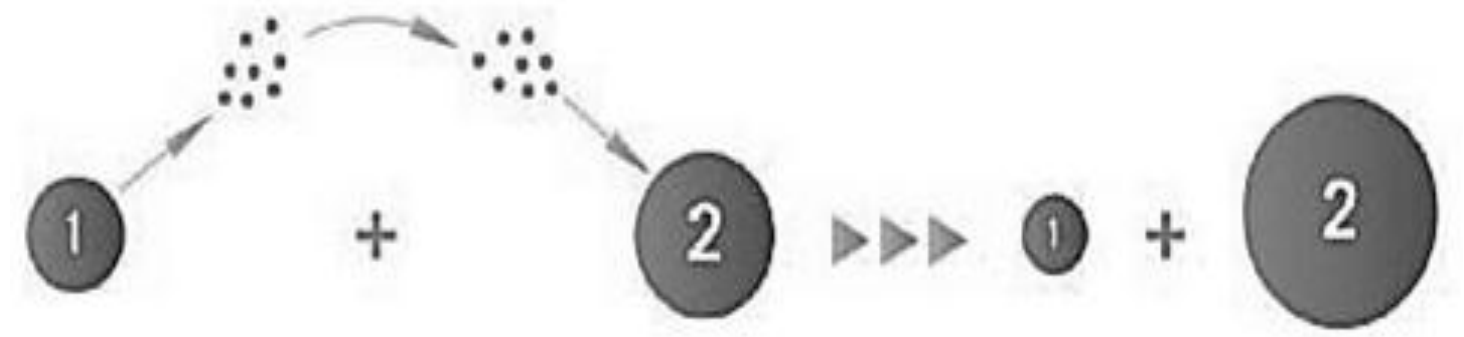

B. Stabilized particle: smaller particle with higher radial pressure evaporates more molecules into the medium and larger particles with smaller radial pressure condenses molecules from medium

Figure 4: Process of Ostwald ripening (4A \& 4B)

For dispersions, a critical parameter that determines if Ostwald ripening will occur is the solubility of the particular material in whatever liquid is chosen. Ostwald ripening only occurs for the substances that are sparingly soluble (BCS Class II/IV drugs); it does not occur if the substances are either completely soluble or completely insoluble. The consequence of Oswald ripening of suspension is that larger particles grow at the expense of the smaller ones. This increase on size can occur via two mechanisms. The first is that small particles 'dissolve' but because the solubility product is low, once sufficient material has dissolved and saturation is achieved any further dissolution results in nucleation followed by precipitation onto larger particles. The second mechanism occurs at higher particle concentrations. In this case, the smaller particles simply aggregate directly onto the surface of the larger particles which is thermodynamically, the preferred route. Thus Ostwald ripening is both solubility and concentration dependent and the rate of ripening is also depends on the viscosity of the suspending medium.

\section{CONCLUSION:}

This review elaborates the steps involved in the formulation of the particulate disperse systems. Knowledge of this process together with an understanding of the physical and chemical properties of the API is necessary to formulate pharmaceutically acceptable particulate disperse system. The aim is to tailor the resulting formulation to be stable during its entire shelf life and produce the desired pharmacological effect without any compromise. The formulation parameters that can be sightseen include particle size, surface charge or zeta potential and surface coating. Selection of wetting agent, deagglomerating agent, stabilizers and their concentration also plays an important role in the dispersion process and subsequently in the formulation of a stable product with adequate shelf life. Proper sequence of the different steps of the dispersion process is essential to produce a stable product, alteration in the sequence of the steps can lead to de-stabilization of the particulate suspension.

\section{ACKNOWLEDGEMENT:}

Authors thankful to the Prof. (Dr.) S. J. Surana and Dr. (Mrs.) V. S. Belgamwar for their valuable suggestion and support during preparation of this review article.

\section{CONFLICT OF INTEREST:}

The authors report no conflicts of interest. The authors alone are responsible for the content and writing of this article. 


\section{REFERENCES:}

1. Slowson M, Slowson S, What to do when patient can't swallow their medications, Pharmacy Times, 1985, 90-96.

2. Lindgren S, Janzon L, Prevalence of swallowing complaints and clinical finding among 50 to 79 year old men and women in urban population, Dysphasia, 1991,6,187-192.

3. Doheny K, You really expect me to swallows those horse pills?Am Druggist, 1993,208,34-35.

4. Ali S, Quadir A, High molecular weight povidone polymer based film for fast dissolving drug delivery applications, Drug Del. Tech. 2007,7(6),36-43.

5. Muller RH, Jacobs C, Kayser O,Nanosuspension as particulate drug formulations in therapy: rationale for development and what we expect for the future, Adv. Drug Del. Rev. 2001, 47(1),3-11.

6. Patel D, Sawant KK, Oral bioavailability enhancement of acyclovir by self-microemulsifying drug delivery systems (SMEDDS), Drug Dev. Ind. Pharm. 2007,33(12),13-18.

7. Attia IA, El-Gizawy SA, Fouda MA, Donia AM, Influence of a niosomal formulation on the oral bioavailability of acyclovir in rabbits, AAPS Pharm. Sci. Tech. 2007,8(4),E106.

8. Kesisoglou F, Panmai S, Wu Y,Nanosizing oral formulation development and biopharmaceutical evaluation, Adv. Drug Del. Rev. 2007,59(7),631-634.

9. Vyas TK, Sahiwala A, Amiji MM, Improved bioavailability and brain transport of saquinavir upon administration in novel nanoemulsion formulations, Int. J. Pharm. 2008,347(1-2),93-96.

10. Takagi T, Ramchandran C, Bermejo M, Yamashita S, Yu LX, Amidon GL, A provisional biopharmaceutical classification of the top 200 oral drug products in United States, Great Britain and Japan, Mol. Pharm. 2006,3(6),631-643.

11. Kasim NA et al, Molecular properties of WHO essential drugs and provisional biopharmaceutical classification, Mol. Pharm. 2004,1(1),85-96

12. Lawrence SG. Dispersions of powder in liquids, $3{ }^{\text {rd }}$ Ed., London: Applied Science Publishers; 1981. P. 1026-1032.

13. Lobenberg R, Amidon GL, Modern bioavailability, bioequivalence and biopharmaceutical classification system: new scientific approaches to international regulatory standards, Eur. J. Pharm. Biopharm. 2000,50(1),3-12.

14. Fairhurst D, Lee RW, Aggregation, agglomeration - how to avoid aggravation when formulating particulate suspensions, Drug Del. Tech., 2008, 8(8), 48-52.

15. Hunter RJ. Zeta potential in colloid science, London: Academic Press; 1981. P. 213-219.

16. Fleer GJ, Stuart MA. Polymers at interface, London: Chapman \& Hall; 1993. P. 93-98.

40.
17. Liu R.Water insoluble drug formulations, $2^{\text {nd }}$ Ed. Boca Raton: CRC Press; 2008. P.149-355

18. Allen T. particle size measurement, $4^{\text {th }}$ Ed., New York: Chapman \& Hall; 1990. P. 111-119.

19. Weiner BB, Particle size distribution - assessment and characterization, American Chemical Society; 1987, 332, 123 125.

20. Hunter RJ. Foundation of colloidal sciences, New York: Oxford University Press; 1987. P. 344-351.

21. Buckley HE. Crystal growth, New York: John Willey; 1951. P. 165-171.

22. Lyklema J. fundamentals of interface and colloidal science, Amsterdam: Elsevier; 1991. P. 235-239.

23. Burgess DJ. Injectable dispersed systems: formulation, processing and performance, New York: Taylor \& Francis; 2005. P. 355-360.

24. Stein HN.The preparation of dispersion in liquids, New York: Marcel Dekker; 1996. P. 356-362.

25. Eirich FR. Rheology: theory and application, Vol. I, New York: Academic Press; 1956. P. 654-659.

26. Goodwin J, Hughes R. Rheology for chemist - An introduction, Cambridge: RSC Publications; 2000. P. 459-471.

27. http://www.elan.com/EDT/nanocrystal_technology

28. http://www.skyepharma.com/solubilzation_idd.html

29. http://www.eurand.com/Technologies/Bioavailabilityenhancement/Biorise

30. http://www.baxterbiopharmasolutions.com/content/formulation_t ech/nanoedge.html

31. http://www.nanotechproject.org/inventories

32. http://www.marks-clerk.com/nanotechnology-report

33. Everett DH, Basic principles of colloidal sciences, London: RSC Publications; 1998. P. 153-156.

34. Fairhurst D, Mitchnik MA. Fundamentals of particles in liquid dispersion, $2^{\text {nd }}$ Ed. New York: Marcel Dekker; 1997. P. 11361141.

35. Evans DF, Wennerstrom H. The colloidal domain, New York: VCH Publishers; 1994. P. 123-129.

36. Washburn EW, The dynamics of capillary flow, Physical Rev. 1921, 17, 273-279.

37. Nancollas GH, The growth of crystals in solution, Adv. Coll. Interface Sci. 1979, 10, 215-221.

38. Higuchi WI, Misra J, Physical degradation of emulsions via the molecular diffusion route and the possible prevention thereof, J. Pharm. Sci. 1962,51(5),459-463.

39. Painter P, Coleman MM. fundamental of polymer science, Lancaster: Technomic Publishing Co.; 1994. P. 389-394. 